\title{
The Balance between Receptor Recycling and Trafficking toward Lysosomes Determines Synaptic Strength during Long-Term Depression
}

\author{
Mónica Fernández-Monreal, ${ }^{1}$ Tyler C. Brown, ${ }^{2}$ María Royo, ${ }^{1}$ and José A. Esteban ${ }^{1}$ \\ ${ }^{1}$ Centro de Biología Molecular "Severo Ochoa," Consejo Superior de Investigaciones Científicas, Madrid 28049, Spain, and 2Department of Pharmacology, \\ University of Michigan Medical School, Ann Arbor, Michigan 48109
}

\begin{abstract}
The strength of excitatory synaptic transmission depends partly on the number of AMPA receptors (AMPARs) at the postsynaptic surface and, thus, can be modulated by membrane trafficking events. These processes are critical for some forms of synaptic plasticity, such as long-term potentiation and long-term depression (LTD). In the case of LTD, AMPARs are internalized and dephosphorylated in response to NMDA receptor activation. However, the fate of the internalized receptors upon LTD induction and its relevance for synaptic function is still a matter of debate. Here we examined the functional contribution of receptor recycling versus degradation for LTD in rat hippocampal slices, and their correlation with receptor dephosphorylation. We observed that GluA1 undergoes sequential dephosphorylation and degradation in lysosomes after LTD induction. However, this degradation does not have functional consequences for the regulation of synaptic strength, and therefore, for the expression of LTD. In contrast, the partition of internalized AMPARs between Rab7-dependent trafficking (toward lysosomes) or Rab11dependent endosomes (recycling back toward synapses) is the key factor determining the extent of synaptic depression upon LTD induction. This sorting decision is related to the phosphorylation status of GluA1 Ser845, the dephosphorylated receptors being those preferentially targeted for lysosomal degradation. Altogether, these new data contribute to clarify the fate of AMPARs during LTD and emphasize the importance of membrane sorting decisions to determine the outcome of synaptic plasticity.
\end{abstract}

\section{Introduction}

AMPA-type glutamate receptors (AMPARs) are responsible for most synaptic transmission at excitatory synapses. These receptors can be inserted or removed from synapses in response to neuronal activity, leading to long-lasting changes in synaptic strength, such as long-term potentiation or long-term depression (LTD) (Malenka and Bear, 2004). Subunit composition, phosphorylation state of AMPAR subunits, and interaction with several scaffolding proteins are important for AMPAR localization, stabilization, and membrane trafficking (Sheng and Lee, 2001; Malinow and Malenka, 2002). This complex regulation suggests highly structured dynamics in the intracellular transport of these receptors. Indeed, endosomal trafficking is important for the synaptic sorting of AMPARs, whose functional compartmentalization is governed by multiple GTPases of the Rab family (Gerges et al., 2004; Brown et al., 2005, 2007). In particular, dur-

Received Jan. 5, 2012; revised July 3, 2012; accepted July 31, 2012.

Author contributions: M.F.-M., T.C.B., and J.A.E. designed research; M.F.-M. and T.C.B. performed research; M.R. contributed unpublished reagents/analytic tools; M.F.-M. and T.C.B. analyzed data; M.F.-M. and J.A.E. wrote the paper.

This work was supported by grants from the Spanish Ministry of Science and Innovation (SAF-2008-04616, SAF-2009-05558-E, CSD-2010-00045 and SAF-2011-24730), Fundación Ramón Areces and Institute de France-NRJ to J.A.E., and by the International Reintegration Grant from the European FP7 to M.F.-M. M.R. and M.F.-M. are supported by the Spanish National Research Council. We thank Marino Zerial for the Rab7 constructs.

Correspondence should be addressed to José A. Esteban, Centro de Biología Molecular "Severo Ochoa," Nicolás Cabrera 1, Madrid 28049, Spain. E-mail: jaesteban@cbm.uam.es.

T. C. Brown's present address: Department of Neuroscience, Brown University, Providence, Rhode Island 02912. DOI:10.1523/JNEUROSCI.0061-12.2012

Copyright $\odot 2012$ the authors $\quad 0270-6474 / 12 / 3213200-06 \$ 15.00 / 0$ ing LTD, AMPARs are internalized by a Rab5-dependent mechanism (Brown et al., 2005). After endocytosis, AMPARs may follow two downstream pathways: recruitment to recycling endosomes for plasma membrane insertion or targeting to late endosomes en route to lysosomes for cargo degradation (Ehlers, 2000; Lin et al., 2000). However, the contribution of these alternative pathways to synaptic plasticity has not been directly evaluated. Thus, although lysosomal localization of AMPARs after LTD induction is well established (Ehlers, 2000; Lee et al., 2004), it is still unknown whether lysosomal-dependent degradation of AMPARs contributes to synaptic depression.

In this work, we show that the GluA1 subunit of AMPARs is degraded in lysosomes in an NMDA receptor (NMDAR)dependent manner upon LTD induction in hippocampal slices. This degradation occurs preferentially on dephosphorylated receptors. However, lysosomal function is not required for LTD. Instead, sorting of internalized AMPARs via Rab7-driven late endosomes favors synaptic depression, whereas Rab11-dependent receptor recycling attenuates LTD expression. Therefore, our work demonstrates that driving AMPAR toward lysosomes is an important event for LTD as a membrane sorting decision, separable from the final enzymatic degradation of the receptor.

\section{Materials and Methods}

Material. Chemicals were purchased from Sigma-Aldrich when not specified otherwise. AMPA and DL-APV were obtained from Tocris Biosciences. GluA1 antibodies were purchased from Abcam (intracellular epitope) and Millipore (surface epitope); antibody phospho-specific for Ser845 of GluA1 was from Affinity Bioreagents. 

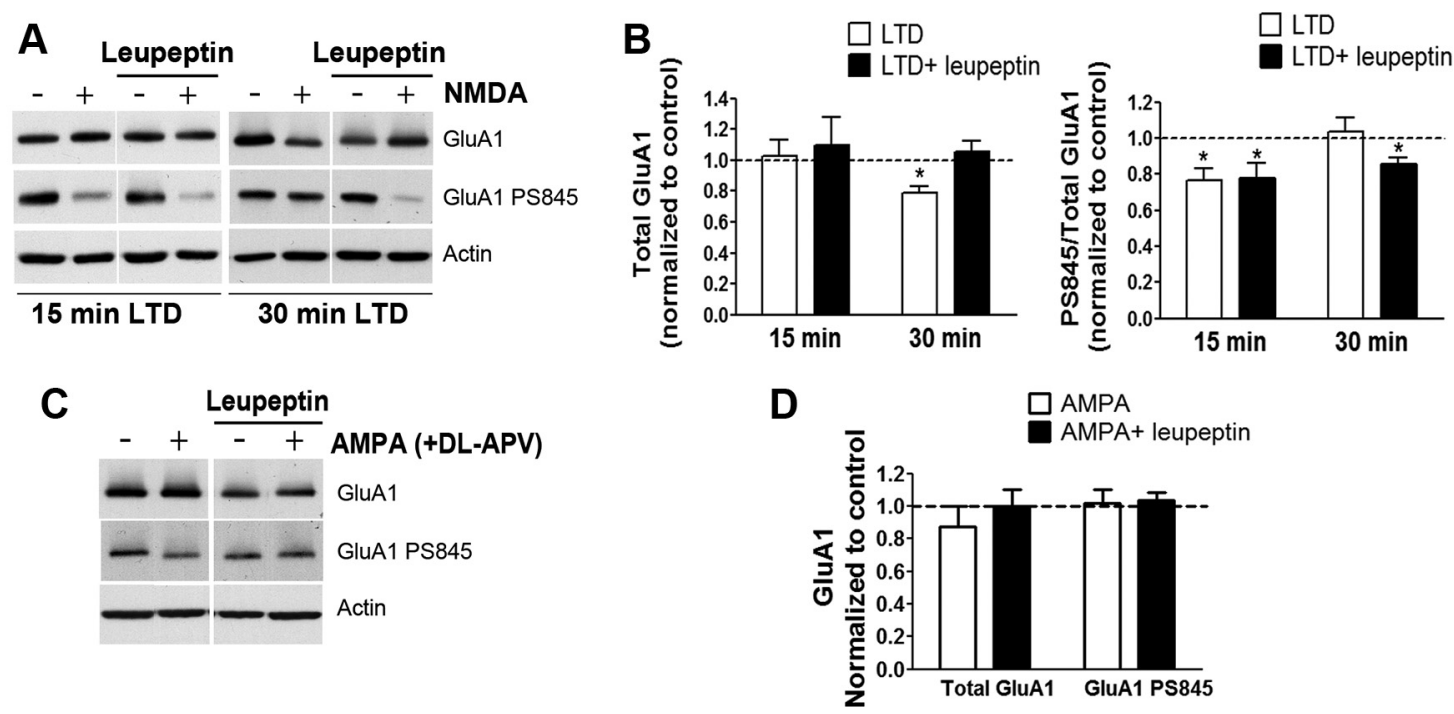

Figure 1. AMPA receptors are degraded upon chemical LTD in lysosomes. $\boldsymbol{A}$, Hippocampal slices were incubated with $20 \mu \mathrm{M}$ NMDA for 5 min (LTD) and protein extracts were analyzed by immunoblotting at different times (left, $15 \mathrm{~min}$; right, $30 \mathrm{~min}$ ) after treatment. Leupeptin (100 $\mu \mathrm{g} / \mathrm{ml}$ ) was added in some slices $4 \mathrm{~h}$ before the experiment and was present during the NMDA treatment. $\boldsymbol{B}$, Immunoblotting quantification for total GluA1 subunit and for phosphorylated GluA1 ( $n=8$ for 15 min and $n=4$ for 30 min; * significantly different from control, $p<0.05$ ). C, Hippocampal slices were treated with $100 \mu \mathrm{M}$ AMPA plus NMDA receptor inhibitor DL-APV (100 $\mu \mathrm{m})$ for 5 min, and analyzed by immunoblotting at 30 min after treatment. $\boldsymbol{D}$, Quantification of total GluA1 and phosphorylated GluA1 $(n=4)$.

Hippocampal slice cultures. Organotypic cultures were prepared from young Wistar rats of either sex (postnatal day 5-6) and placed on semiporous membranes for 4-7 d. Expression of single recombinant proteins was performed with the Sindbis virus expression system (Malinow et al., 1999).

Electrophysiology. The recording chamber was perfused with the following (in mM): $119 \mathrm{NaCl}, 2.5 \mathrm{KCl}, 4 \mathrm{CaCl}_{2}, 4 \mathrm{MgCl}_{2}, 26 \mathrm{NaHCO}_{3}, 1$ $\mathrm{NaH}_{2} \mathrm{PO}_{4}, 11$ glucose, 0.1 picrotoxin, and 0.004 2-chloroadenosine, $\mathrm{pH}$ 7.4 , gassed with $5 \% \mathrm{CO}_{2} / 95 \% \mathrm{O}_{2}$. Patch recording pipettes (3-6 $\mathrm{M} \Omega$ ) were filled with the following (in $\mathrm{mM}$ ): 115 cesium methanesulfonate, 20 CsCl, 10 HEPES, $2.5 \mathrm{MgCl}_{2}, 4 \mathrm{Na}_{2} \mathrm{ATP}, 0.4 \mathrm{Na}_{3} \mathrm{GTP}, 10$ sodium phosphocreatine, and 0.6 EGTA, pH 7.25. Synaptic responses were evoked with bipolar electrodes using single-voltage pulses (200 $\mu$ s, up to $20 \mathrm{~V})$. LTD was induced using a pairing protocol by stimulating Schaffer collateral fibers at $1 \mathrm{~Hz}$ (300 pulses) while depolarizing the postsynaptic cell to $-40 \mathrm{mV}$.

Hippocampal primary cultures. Hippocampi were dissected from E18 rat embryos and cells dissociated with trypsin. Neurons were plated onto poly-L-lysine-coated coverslips and cultured in Neurobasal medium supplemented with B27 and glutamine. Cells were maintained at $37^{\circ} \mathrm{C}$ and $5 \% \mathrm{CO}_{2}$. Neurons were infected with Sindbis virus driving the expression of EGFP or Rab7-DN for $24 \mathrm{~h}$.

Extracellular staining of surface AMPA receptors and image analysis. Neuronal cultures were washed and maintained for $30 \mathrm{~min}$ in an extracellular solution containing the following (in mM): $150 \mathrm{NaCl}, 2 \mathrm{CalCl}_{2}, 5$ $\mathrm{KCl}, 10$ HEPES (pH 7.4), 30 glucose, and 0.001 tetrodotoxin. Neurons were untreated or treated for 5 min with $20 \mu \mathrm{M}$ NMDA plus $1 \mu \mathrm{M}$ glycine, and then fixed at different times with $4 \%$ paraformaldehyde plus $4 \%$ sucrose for $15 \mathrm{~min}$ on ice. Surface AMPA receptors were stained with an antibody directed against the $N$-terminal epitope of GluA1 (Millipore) without permeabilization. Images were acquired by laser-scanning confocal microscopy. Total fluorescence intensity from the maximum Z-projection was determined by ImageJ and normalized to total area of the neuron. Data presented are means \pm SEMs.

GST pull-down. Rab11-FIP2 was fused downstream from GST, expressed in BL21 star bacteria, and loaded onto glutathione beads (GE Healthcare). Pull-down controls were performed with $0.1 \mathrm{~mm}$ nonhydrolyzable GTP analog (GMP-PNP) or 1 mM GDP. Hippocampal extracts were prepared in homogenization buffer containing the following (in mM): 10 HEPES, $150 \mathrm{NaCl}, 5 \mathrm{MgCl}_{2}, 1 \mathrm{DTT}, 1 \%$ Triton X-100, and $5 \%$ glycerol. Extracts from each condition were incubated with GST beads for $1.5 \mathrm{~h}$ at $4^{\circ} \mathrm{C}$, followed by three washes in homogenization buffer.

Statistical analyses. All graphs represent average values \pm SEM. Statistical differences were calculated according to nonparametric tests. When significant differences were observed, $p$ values for pairwise comparisons were calculated according to two-tailed Mann-Whitney tests (for unpaired data) unless otherwise noted, or Wilcoxon's tests (for paired data).

\section{Results}

Sequential dephosphorylation and lysosomal degradation of AMPARs upon LTD

Most of the evidence on lysosomal-dependent degradation of AMPARs during LTD has been obtained in primary neuronal cultures (Ehlers, 2000; Lee et al., 2004). Therefore, we started by investigating the degradation of the GluAl subunit of AMPARs during long-term depression in hippocampal slices. To this end, we used a chemical protocol of LTD (c-LTD, 20 $\mu \mathrm{M}$ NMDA, $5 \mathrm{~min}$ ) that maximizes the number of synapses undergoing plasticity (Lee et al., 1998). We analyzed protein content and phosphorylation state by immunoblotting at different times after NMDA application. As shown in Figure 1, $A$ and $B$, treatment with NMDA induced a significant degradation of GluA1 at $30 \mathrm{~min}$, which is blocked by the inhibitor of lysosome degradation leupeptin $(100 \mu \mathrm{g} / \mathrm{ml})$. This degradation is delayed in time, since it is not observable at $15 \mathrm{~min}$. As dephosphorylation of the Ser845 of the GluA1 subunit is a hallmark of LTD (Lee et al., 1998), we monitored GluA1 phosphorylation in correlation with receptor degradation. We observed that this subunit is significantly dephosphorylated at 15 min, independent from lysosomal degradation. This result suggests that AMPAR dephosphorylation is an early event after c-LTD induction. Interestingly, GluA1 dephosphorylation is detectable at $30 \mathrm{~min}$ after c-LTD only if lysosomal degradation is blocked with leupeptin (Fig. $1 A, B$ ). This suggests that dephosphorylated receptors are preferentially targeted for degradation. Hence, GluA1 dephosphorylation at Ser845 appears to be a tag for lysosomal degradation. 
Next, we tested whether dephosphorylation and degradation of GluA1 were specific for NMDAR activation, since direct activation of AMPARs (with AMPA) has also been shown to trigger receptor internalization (Ehlers, 2000). As shown in Figure 1, $C$ and $D$, treatment with AMPA $(100 \mu \mathrm{M}, 5 \mathrm{~min}$, in the presence of $100 \mu \mathrm{M}$ DL-APV to prevent NMDAR activation) neither induced degradation nor dephosphorylation of GluA1 at $30 \mathrm{~min}$ of treatment. Altogether, these results suggest that during LTD, the GluA1 subunit of AMPAR undergoes an early dephosphorylation, which is correlated to the subsequent degradation of the receptor by lysosomes. These events are triggered by NMDAR activation, and are not a necessary consequence of ligand-induced receptor internalization.

\section{Lysosomal degradation is not necessary} for LTD expression

We tested whether AMPAR lysosomal degradation was important for synaptic plasticity using electrophysiological recordings. We induced LTD in the CA1 region of the hippocampus by stimulating Schaffer collaterals for $5 \mathrm{~min}$ at $1 \mathrm{~Hz}$. This protocol produces a decrease in the EPSCs of $\sim 40 \%$ from the baseline (Fig. $2 A, B$, Vehicle). Importantly, incubation of the slices with leupeptin (for 4 or $24 \mathrm{~h}$, as indicated) had no effect on LTD (Fig. $2 A, B$; leupeptin was also present during the recordings), although these conditions were effective to suppress lysosomal-dependent degradation of AMPARs (Fig. $1 A, B$ ).

In addition, we tested c-LTD at electrophysiological level. As shown in Figure 2, $C$ and $D$, no differences were found between slices treated and untreated with leupeptin during c-LTD. These results are virtually identical to those obtained with synaptic induction of LTD (Fig. 2A,B). Therefore, these combined data suggest that lysosomal degradation of AMPARs is not necessary for the functional expression of synaptic depression in hippocampal slices.

\section{Membrane sorting toward lysosomes or recycling endosomes determines LTD expression}

Targeting of membrane proteins for lysosomal degradation requires endosomal sorting mediated by small GTPases of the Rab family. Therefore, we explored whether the trafficking of AMPARs to lysosomes was important for LTD. For this purpose, we used a dominant negative (DN) version of the small-GTPase Rab7, which mediates the trafficking of target proteins to lysosomes from late-endosomes (Press et al., 1998). As shown in Figure 3, $A$ and $B$, blockade of Rab7-dependent trafficking produced a significant reduction in LTD expression compared with control (uninfected) neurons. This result suggests that Rab7driven trafficking of AMPARs to lysosomes is important for LTD expression.

We reasoned that recycling endosomes may constitute an alternative pathway for internalized AMPARs after LTD induction, as opposed to trafficking toward lysosomes. To evaluate the contribution of this pathway for synaptic depression, we induced
LTD on CA1 hippocampal neurons expressing a dominant negative version of Rab11 (Rab11-DN), which blocks the transport of AMPARs from recycling endosomes to the postsynaptic compartment (Park et al., 2004; Brown et al., 2007). We observed a significant increase in LTD expression, compared with control neurons (Fig. 3C,D). This result suggests that a fraction of the internalized AMPARs upon LTD induction return to the synaptic membrane via Rab11-driven recycling endosomes.

Finally, we tested whether the overexpression of Rab7-DN affects basal transmission in hippocampal neurons. Simultaneous recordings were obtained from nearby pairs of CA1 neurons expressing Rab7-DN and control (uninfected) neurons. As shown in Figure 3, $E$ and $F$, overexpression of Rab7-DN does not affect synaptic responses mediated by AMPA or NMDA receptors. Similar results were previously obtained with Rab11-DN-expressing neurons (Gerges et al., 2004). Therefore, semiacute $(18-24 \mathrm{~h})$ blockade of recycling (Rab11) or late (Rab7) endosomal trafficking does not alter basal excitatory synaptic transmission.

An important question about the endolysosomal trafficking of AMPARs during LTD is whether it is specific to the activation of synaptic or extrasynaptic NMDARs. The pharmacological approach used in Figure 1 was used to maximize the number of synapses undergoing plasticity and, therefore, increase the sensitivity to detect biochemical changes. We wanted to evaluate whether c-LTD displays the same dependence on Rab proteins as the synaptically induced one. As shown in Figure $4, A-D$, this was the case for both Rab7 and Rab11: Rab7-DN attenuates c-LTD and Rab11-DN enhances it. These data reinforce the interpreta- 

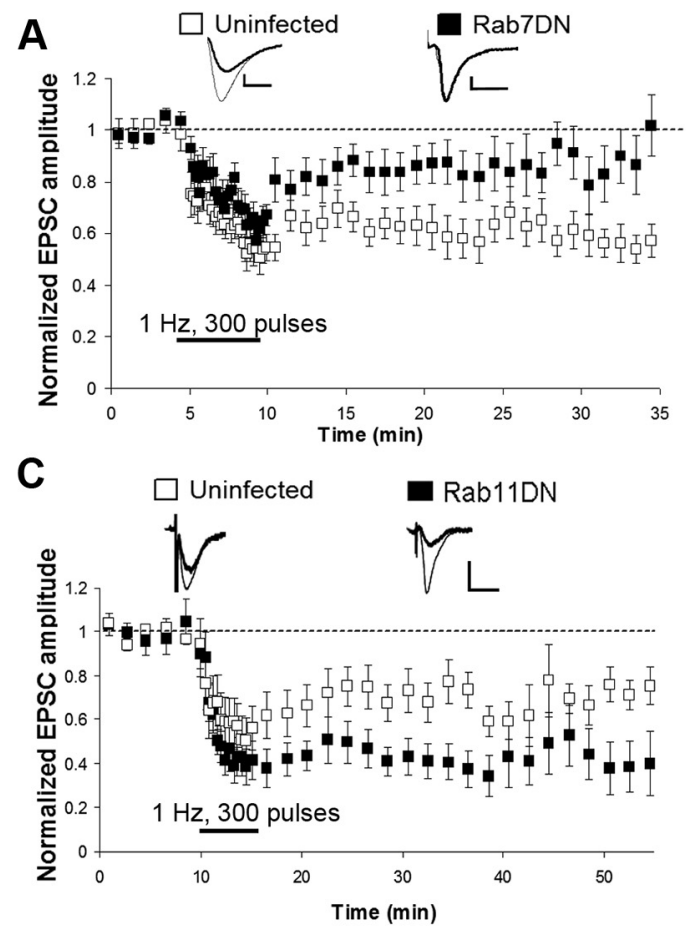

E

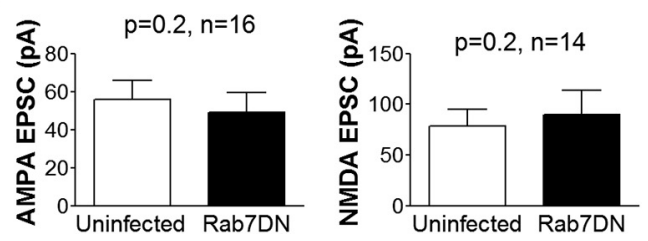

B

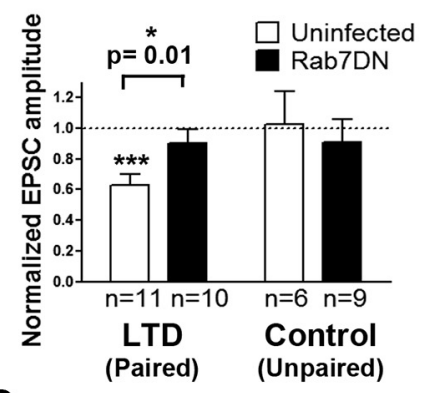

D

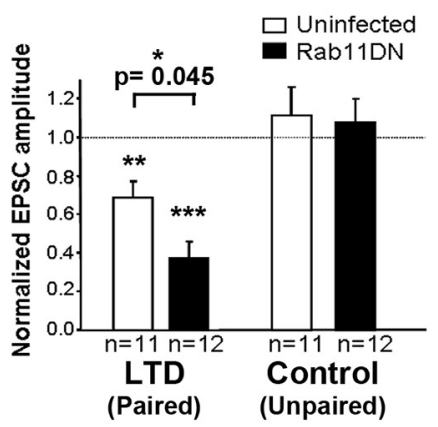

$F$

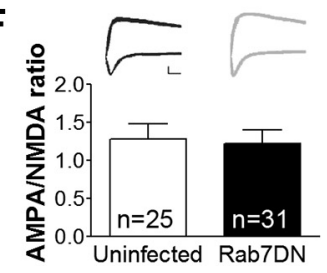

Figure 3. Degradation and recycling pathways of AMPA receptors are involved in LTD expression. A, LTD was induced in slices infected with Sindbis virus expressing the DN version of Rab7 or from uninfected slices. Amplitude of the synaptic responses is normalized to a 5 min baseline. Scale bars: vertical, $20 \mathrm{pA}$; horizontal, $5 \mathrm{~ms}$. B, Average of AMPA receptormediated responses collected from the last $5 \mathrm{~min}$ of the recording and normalized to the baseline. Left bars (LTD, paired) correspond to the stimulation pathway in which postsynaptic depolarization $(-40 \mathrm{mV})$ was paired to low-frequency stimulation (***significantly different from baseline, $p<0.005$ ). Right bars (control, unpaired) correspond to the pathway that was not stimulated during depolarization. $\boldsymbol{C}$, Same LTD experiment as in $\boldsymbol{A}$ from neurons infected with Sindbis virus expressing Rab11-DN or control (uninfected) neurons. Amplitude of the synaptic responses is normalized to a $10 \mathrm{~min}$ baseline. Scale bars: vertical, $20 \mathrm{pA}$; horizontal, $30 \mathrm{~ms}$. A, C, Insets, Sample traces averaged from baseline (thin lines) or from the last $5 \mathrm{~min}$ of the recording (thick lines). $D$, Average of AMPA receptor-mediated responses collected from the last $5 \mathrm{~min}$ of the recording and normalized to the baseline. Left bars (LTD, paired) correspond to the stimulation pathway ( ${ }^{*}$ significantly different from baseline, $p<0.05 ;{ }^{* *}$ significantly different from baseline, $p<0.01$ ). $\boldsymbol{E}$, Average of AMPA receptor- (left) and NMDA receptor- (right) mediated current amplitude (recorded at $-60 \mathrm{mV}$ and $+40 \mathrm{mV}$, respectively) from pairs of uninfected and Rab7DN-expressing neurons. $F$, Comparison of evoked synaptic responses from uninfected and infected cells. AMPA/NMDA ratio is calculated from the size of the AMPAR- and NMDAR-mediated responses. Insets, Representative traces. Scale bars: vertical, $50 \mathrm{pA}$; horizontal, $10 \mathrm{~ms}$.

tion that these two protocols of LTD induction are functionally equivalent, at least with respect to Rab-dependent trafficking and lysosomal degradation.

Blocking Rab7-dependent trafficking increase the recycling of AMPA receptors to surface

Our electrophysiological results suggest that after blocking the degradation pathway, internalized AMPARs are recycled back to the surface. To test this possibility, we monitored surface AMPARs in hippocampal neurons during LTD upon suppression of Rab7-dependent trafficking. As shown in Figure 4, E and F, there were no significant differences in the amount of surface receptors between EGFP and Rab7-DN-expressing neurons under basal conditions. In contrast, as shown in Figure 4, E and $G$, upon NMDA treatment, Rab7-DN expression increases AMPAR surface levels at 15 and $30 \mathrm{~min}$. These results are in agreement with the electrophysiological recordings from Figures $3 A$ and $4 A$. These combined data are consistent with our interpretation of Rab7-DN blocking endolysosomal trafficking of internalized receptors, and therefore, favoring their recycling to the neuronal surface. Alternatively, it is also possible that Rab7$\mathrm{DN}$ attenuates receptor endocytosis at late time points during LTD due to a general alteration in early endosomal trafficking.

\section{Rab11 activity levels are high and do not change during LTD}

Finally, we determined whether Rab11 activity is altered during the recycling events taking place during LTD. To monitor the levels of active (GTP-bound) Rab11 during LTD induction, we designed a GST pull-down assay using the Rab11 effector protein Rab11-Family Interacting Protein2 (FIP2), which specifically binds Rab11-GTP (Junutula et al., 2004). To evaluate the specificity of the GST fusion protein, we preloaded hippocampal extracts with a nonhydrolyzable GTP analog or with GDP, followed by incubation with beads containing either GST alone or GST fused to FIP2 (GST-FIP2) (see Materials and Methods). As shown in Figure $4 H$ (top), GST-FIP2 preferentially binds Rab11-GTP, with only a weak binding to Rab11-GDP. Interestingly, the amount of Rab11 pulled-down from untreated extracts (without nucleotide preloading) was comparable to the condition with nonhydrolyzable GTP, suggesting that hippocampal extracts contain high levels of active Rab11 under basal conditions.

We then evaluated Rab11 activation during LTD. We found that the amount of active (GTP-bound) Rab11 is not altered relative to untreated, control slices (Fig. $4 H$, middle). These results imply that basal Rab11 activity is sufficient to support the recycling of newly internalized AMPA receptors back to the neuronal surface after LTD induction.

\section{Discussion}

These studies present a functional dissection of the postendocytic trafficking pathways of AMPARs and how the choice between receptor recycling and lysosomal targeting determines the extent of synaptic depression during LTD. Thus, a fraction of internalized AMPARs are driven via Rab11-dependent recycling endosomes back to the synaptic membrane, and therefore, this process contributes to attenuate synaptic depression. Conversely, some AMPARs traffic with Rab7-dependent late endosomes toward lysosomes. When this pathway is blocked (Rab7-DN), receptor recycling is favored and synaptic depression is decreased (although we cannot rule out an indirect impairment of receptor 

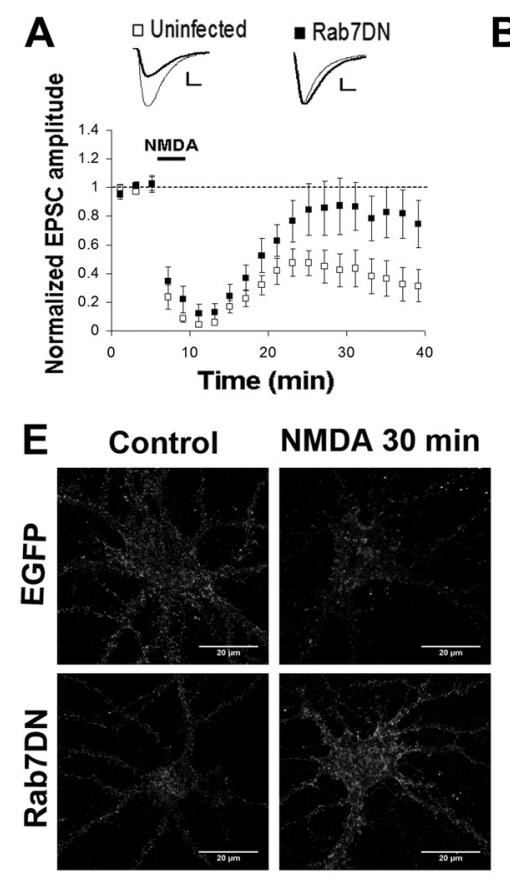
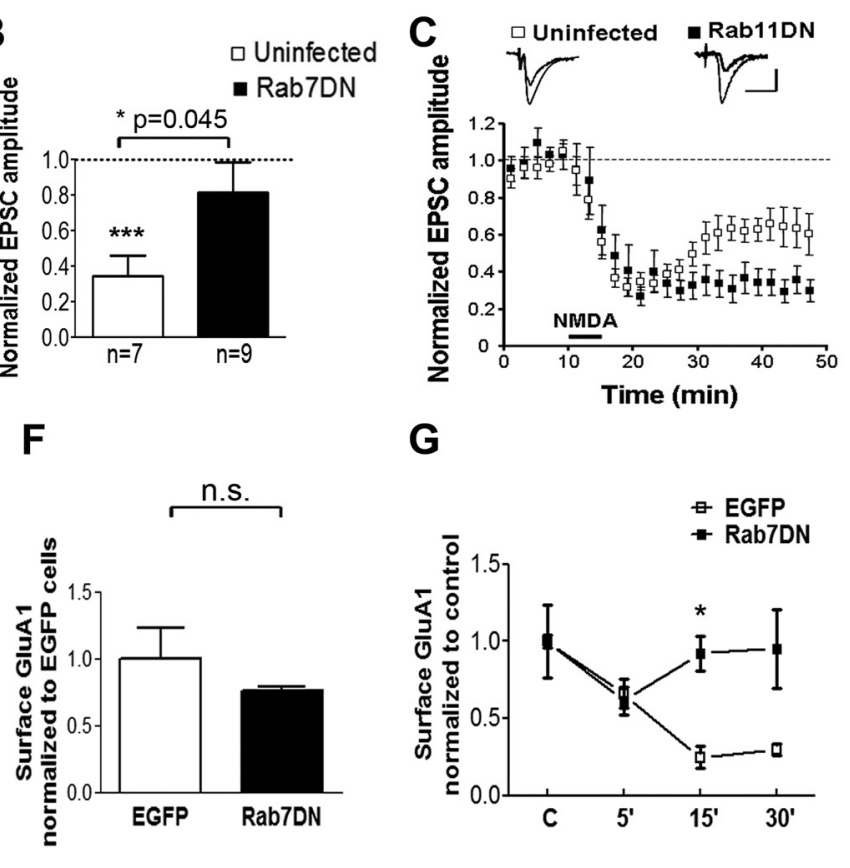

G

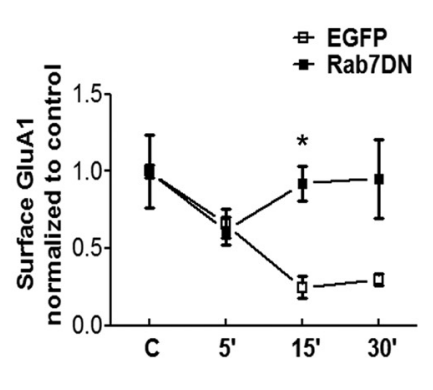

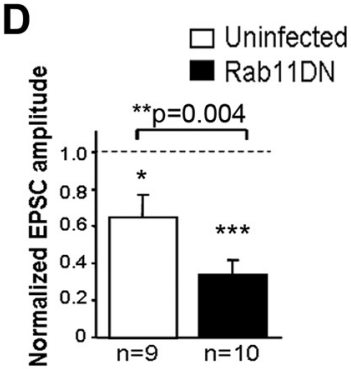

H

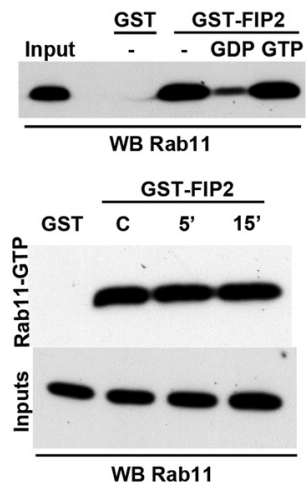

Figure 4. Blocking Rab7-dependent trafficking to lysosomes increases recycling of AMPA receptors to the surface. A, C, LTD was chemically induced by bath incubation with NMDA (20 $\mu \mathrm{M}$ ) for 5 min in control slices and slices infected with Rab7-DN (A) or with Rab11-DN (C). Amplitude of synaptic responses was normalized to a 5 min baseline. Insets, Sample traces averaged from the baseline (thin lines) or from the last $5 \mathrm{~min}$ of the recording (thick lines). Scale bars: vertical, $20 \mathrm{pA}$; horizontal, $5 \mathrm{~ms}$. B, D, Average of AMPA receptor response collected from the last 5 min of the time course and normalized to the baseline from the recordings shown in $\boldsymbol{A}$ and $\boldsymbol{C}$ (significantly different from baseline: ${ }^{* *} p<0.01,{ }^{*} p<0.05$ ). $\boldsymbol{E}$, Representative images of surface GluA1 immunostaining of hippocampal neurons infected with Sindbis virus driving the expression of EGFP or Rab7-DN, untreated (control) or treated with $20 \mu \mathrm{m}$ NMDA for 5 min plus 30 min of washout. $\boldsymbol{F}$, Quantification of surface AMPA receptors under basal conditions from neurons infected with virus expressing EGFP or Rab7-DN. G, Time course quantification of surface GluA1 from neurons infected with virus expressing EGFP or Rab7-DN during c-LTD. *Significantly different from EGFP infected neurons, $p<0.05$. $\boldsymbol{H}$, Top, Western blot of Rab11 pulled-down with GST or GST fused to FIP2 (GST-FIP2) from hippocampal extracts. Extracts were preloaded with nonhydrolyzable GTP or with GDP, or left untreated, as indicated. Ten percent of the extract was loaded as reference (left lane, input). Middle, Analysis of Rab11 pulled-down with GST-FIP2.Extracts from some untreated slices were pulled-down with GST alone (left lane). Bottom, Ten percent of the total extracts corresponding to the middle panel. C: Untreated neurons; 5', 15', 30': minutes after NMDA treatment.

endocytosis with Rab7-DN due to a general alteration of early endosomal trafficking). Interestingly, although a fraction of AMPARs are effectively degraded at lysosomes after LTD induction, this enzymatic degradation is not required for depression. This suggests that transport of AMPARs via Rab7 is a no-return pathway that ensures receptor removal from the synaptic membrane, and therefore synaptic depression, regardless from lysosomal action.

Whether AMPARs are degraded after activity-dependent endocytosis and its role in LTD have been controversial questions the last decade. Using primary neuronal cultures, it has been reported that AMPARs follow alternative sorting pathways depending on whether AMPARs or NMDARs are activated (Ehlers, 2000; Lee et al., 2004). In contrast, another report using acute hippocampal slices from adult mice failed to detect AMPA receptor degradation upon pharmacological NMDA receptor activation (Delgado et al., 2007). Our work helps to clarify this issue by determining the relative contribution of recycling versus endolysosomal pathways to synaptic depression. Indeed, our data suggest that AMPARs partition $\sim 50 \%$ into these two pathways after LTD induction, because blockade of receptor recycling approximately doubles synaptic depression, whereas interfering with lysosomal trafficking almost abolishes LTD.

An important corollary of this work is that AMPAR lysosomal degradation is observable after LTD induction in hippocampal slices (but see Delgado et al., 2007). However, we have revealed that lysosome enzymatic action is in fact not relevant for synaptic depression, because leupeptin incubation blocks receptor degra- dation but does not alter LTD expression. In a sense, these results are reminiscent on the role of protein ubiquitination for LTD, where postendocytic trafficking may play the dominant role (Lussier et al., 2011), rather than enzymatic degradation by the proteasome (Citri et al., 2009). Obviously, this interpretation does not preclude a role for ubiquitin-dependent degradation of other proteins in synaptic depression, as it has been described for PSD-95 (Colledge et al., 2003), although this issue remains controversial (Xu et al., 2008; Bhattacharyya et al., 2009).

The existence of a point of choice between receptor recycling and lysosomal trafficking opens the possibility that the relative weight of these two alternative pathways may be modulated to adjust the final outcome of synaptic depression under different circumstances. We have not fully explored this possibility. However, the correlation we have observed between GluAl phosphorylation and receptor degradation does suggest that this point of choice is in fact a regulated one. GluA1 dephosphorylation at Ser845 is a hallmark of AMPAR regulation during LTD (Lee et al., 1998). While monitoring the phosphorylation state of GluA1 during LTD, we observed that receptor dephosphorylation is only detected transiently (at $15 \mathrm{~min}$, but not at $30 \mathrm{~min}$ ), unless lysosomal function was blocked with leupeptin. Therefore, prevention of receptor degradation unmasked an accumulation of dephosphorylated GluA1 after LTD induction. This suggests that dephosphorylated receptors are preferentially degraded by the lysosome, or in other words, dephosphorylation of GluA1 Ser845 appears to be a tag to direct AMPARs to lysosomal degradation during LTD. This is consistent with a previous report where 
phosphomimetic mutations in GluA1 protected AMPAR from lysosomal degradation under basal conditions (Kessels et al., 2009). By controlling the partition between receptor recycling or degradation, protein phosphatases would play a crucial role in determining synaptic strength during LTD. This interpretation provides a mechanistic explanation for the requirement of protein phosphatase activity for LTD as it was originally described (Mulkey et al., 1993).

In summary, this work sheds light on the contribution of postendocytic trafficking of AMPARs to LTD, and shows for the first time that Rab7-dependent transport is required to drive receptors away from recycling endosomes, thereby preventing them from returning to the synaptic membrane.

\section{References}

Bhattacharyya S, Biou V, Xu W, Schlüter O, Malenka RC (2009) A critical role for PSD-95/AKAP interactions in endocytosis of synaptic AMPA receptors. Nat Neurosci 12:172-181.

Brown TC, Tran IC, Backos DS, Esteban JA (2005) NMDA receptordependent activation of the small GTPase Rab5 drives the removal of synaptic AMPA receptors during hippocampal LTD. Neuron 45:81-94.

Brown TC, Correia SS, Petrok CN, Esteban JA (2007) Functional compartmentalization of endosomal trafficking for the synaptic delivery of AMPA receptors during long-term potentiation. J Neurosci 27:13311-13315.

Citri A, Soler-Llavina G, Bhattacharyya S, Malenka RC (2009) N-methyl-Daspartate receptor- and metabotropic glutamate receptor-dependent long-term depression are differentially regulated by the ubiquitinproteasome system. Eur J Neurosci 30:1443-1450.

Colledge M, Snyder EM, Crozier RA, Soderling JA, Jin Y, Langeberg LK, Lu H, Bear MF, Scott JD (2003) Ubiquitination regulates PSD-95 degradation and AMPA receptor surface expression. Neuron 40:595-607.

Delgado JY, Coba M, Anderson CN, Thompson KR, Gray EE, Heusner CL, Martin KC, Grant SG, O'Dell TJ (2007) NMDA receptor activation dephosphorylates AMPA receptor glutamate receptor 1 subunits at threonine 840. J Neurosci 27:13210-13221.

Ehlers MD (2000) Reinsertion or degradation of AMPA receptors determined by activity-dependent endocytic sorting. Neuron 28:511-525.

Gerges NZ, Backos DS, Esteban JA (2004) Local control of AMPA receptor trafficking at the postsynaptic terminal by a small GTPase of the Rab family. J Biol Chem 279:43870-43878.
Junutula JR, Schonteich E, Wilson GM, Peden AA, Scheller RH, Prekeris R (2004) Molecular characterization of Rab11 interactions with members of the family of Rab11-interacting proteins. J Biol Chem 279:3343033437.

Kessels HW, Kopec CD, Klein ME, Malinow R (2009) Roles of stargazin and phosphorylation in the control of AMPA receptor subcellular distribution. Nat Neurosci 12:888-896.

Lee HK, Kameyama K, Huganir RL, Bear MF (1998) NMDA induces longterm synaptic depression and dephosphorylation of the GluR1 subunit of AMPA receptors in hippocampus. Neuron 21:1151-1162.

Lee SH, Simonetta A, Sheng M (2004) Subunit rules governing the sorting of internalized AMPA receptors in hippocampal neurons. Neuron 43:221-236

Lin JW, Ju W, Foster K, Lee SH, Ahmadian G, Wyszynski M, Wang YT, Sheng M (2000) Distinct molecular mechanisms and divergent endocytotic pathways of AMPA receptor internalization. Nat Neurosci 3:1282-1290.

Lussier MP, Nasu-Nishimura Y, Roche KW (2011) Activity-dependent ubiquitination of the AMPA receptor subunit GluA2. J Neurosci 31:3077-3081.

Malenka RC, Bear MF (2004) LTP and LTD: an embarrassment of riches. Neuron 44:5-21.

Malinow R, Malenka RC (2002) AMPA receptor trafficking and synaptic plasticity. Annu Rev Neurosci 25:103-126.

Malinow R, Hayashi Y, Maletic-Savatic M, Zaman SH, Poncer JC, Shi SH, Esteban JA, Osten P, Seidenman K (2010) Introduction of green fluorescent protein into hippocampal neurons through viral infection. Cold Spring Harb Protoc 2010:pdb.prot5406.

Mulkey RM, Herron CE, Malenka RC (1993) An essential role for protein phosphatases in hippocampal long-term depression. Science 261:10511055.

Park M, Penick EC, Edwards JG, Kauer JA, Ehlers MD (2004) Recycling endosomes supply AMPA receptors for LTP. Science 305:1972-1975.

Press B, Feng Y, Hoflack B, Wandinger-Ness A (1998) Mutant Rab7 causes the accumulation of cathepsin D and cation-independent mannose 6-phosphate receptor in an early endocytic compartment. J Cell Biol 140:1075-1089.

Sheng M, Lee SH (2001) AMPA receptor trafficking and the control of synaptic transmission. Cell 105:825-828.

Xu W, Schlüter OM, Steiner P, Czervionke BL, Sabatini B, Malenka RC (2008) Molecular dissociation of the role of PSD-95 in regulating synaptic strength and LTD. Neuron 57:248-262. 